\title{
Violência na Estratégia de Saúde da Família: riscos para a saúde dos trabalhadores e ao atendimento
}

\author{
Violence in the Family Health Strategy: risks to workers' health and care \\ Violencia en la estrategia de salud de la familia: riesgos para la salud de los trabajadores y la atención
}

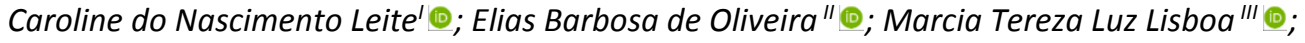 \\ Lucia Helena Garcia Penna "®; Sonia Acioli de Oliveira "®; Ricardo de Matos Russo Rafael"® \\ 'Universidade Estácio de Sá, Rio de Janeiro, RJ, Brasil; "Universidade do Estado do Rio de Janeiro, \\ Rio de Janeiro, RJ, Brasil; Universidade Federal do Rio de Janeiro, Rio de Janeiro, RJ, Brasil
}

\begin{abstract}
RESUMO
Objetivo: identificar a ocorrência da violência na Estratégia de Saúde da Família e analisar as repercussões para a saúde dos trabalhadores e ao atendimento. Método: estudo qualitativo, descritivo, realizado com 27 trabalhadores de uma unidade de Estratégia de Saúde da Família localizada no município do Rio de Janeiro em 2018. Trabalhou-se com a técnica de entrevista semiestruturada mediante roteiro e para a categorização dos depoimentos a análise de conteúdo temática. Projeto de pesquisa aprovado pelo Comitê de Ética em Pesquisa. Resultados: identificou-se a violência psicológica perpetrada por usuários através de ameaças, xingamentos e intimidações e a urbana com a exposição dos profissionais a tiros, assaltos e brigas durante as visitas domiciliares ocasionando repercussões na saúde mental dos trabalhadores e prejuízos para a qualidade do atendimento. Conclusão: a violência ocupacional é um risco a integridade física e psíquica dos trabalhadores e a qualidade do atendimento. Descritores: Estratégia Saúde da Família; Violência no Trabalho; Equipe de Assistência ao Paciente; Saúde Mental.
\end{abstract}

\section{ABSTRACT}

Objective: to identify the occurrence of violence in Brazil's Family Health Strategy and to analyze the repercussions on workers' health and on care. Method: this qualitative, descriptive study was conducted in 2018 with 27 workers from a Family Health Strategy unit in the city of Rio de Janeiro, using the semi-structured, scripted interview technique, and thematic content analysis to categorize interviewee discourse. The project was approved by the research ethics committee. Results: the violence identified was psychological, as perpetrated by users in threats, name calling and intimidation, and urban, in health personnel's exposure to gunfire, robbery, and fights during home visits, which had repercussions on the workers' mental health and impaired the quality of care. Conclusion: occupational violence is a risk to health workers' physical and mental integrity and to the quality of care.

Descriptors: Family Health Strategy; Workplace Violence; Patient Care Team; Mental Health.

\section{RESUMEN}

Objetivo: identificar la ocurrencia de violencia en la Estrategia de Salud de la Familia de Brasil y analizar las repercusiones en la salud y la atención de los trabajadores. Método: este estudio cualitativo descriptivo se realizó en 2018 con 27 trabajadores de una unidad de Estrategia de Salud de la Familia en la ciudad de Río de Janeiro, utilizando la técnica de entrevista semiestructurada, guionizada y análisis de contenido temático para categorizar el discurso del entrevistado. El proyecto fue aprobado por el comité de ética en investigación. Resultados: la violencia identificada fue psicológica, perpetrada por los usuarios en amenazas, insultos e intimidación, y urbana, en la exposición del personal de salud a disparos, robos y peleas durante las visitas domiciliarias, lo que repercutió en la salud mental de los trabajadores y perjudicó la calidad de atención. Conclusión: la violencia laboral es un riesgo para la integridad física y mental de los trabajadores de la salud y para la calidad de la atención.

Descriptores: Estrategia de Salud Familiar; Violencia Laboral; Grupo de Atención ao Paciente; Salud Mental.

\section{INTRODUÇÃO}

A Estratégia Saúde da Família (ESF), como parte da Rede de Atenção à Saúde (RAS) é considerada a principal porta de entrada no Sistema Único de Saúde (SUS), cujo atendimento do usuário tanto no âmbito da unidade quanto em domicílio, inicia-se com o ato de acolher e oferecer resposta resolutiva para os problemas de saúde. O acolhimento com escuta qualificada tem como objetivos o estabelecimento do vínculo e a integralidade do cuidado e, ainda de modo desafiado compreende-se que seja necessário um trabalho multiprofissional e interdisciplinar, com disposição de todos para ouvir e valorizar as trocas e as experiências ${ }^{1}$. 
Um atendimento humanizado contribui para o acesso da população as unidades básicas de saúde, qualifica o trabalho dos profissionais e torna o cuidado mais efetivo, melhorando a saúde de todos os envolvidos e a qualidade de vida 2,3 . É importante considerar que, em geral, existe uma lacuna entre a motivação da busca pelo serviço de saúde e as demandas que possivelmente precisam ser resolvidas pelos profissionais. Ou seja, a relação entre os desejos profissionais e dos usuários nem sempre se estabelecem por interseções, ocasionando conflitos, por vezes, de difícil resolução. A ausência de uma escuta negociadora aliada à cultura da violência estabilizada na sociedade pode caracterizar terreno fértil para a eclosão da violência no trabalho e suas consequências para os profissionais e o cuidado realizado ${ }^{4-6}$.

Acrescenta-se a violência urbana no cotidiano dos serviços da RAS e cujas práticas de enfrentamento não se mostram eficazes, apesar de os profissionais conviverem com as dinâmicas de territórios marcadas pelas altas taxas de homicídios. Tal realidade exige, por parte dos profissionais, intervenções orientadas por uma concepção ampliada de saúde de modo a contemplar os determinantes sociais do processo saúde-doença-cuidado e do cuidado de $\mathrm{si}^{7}$. Neste sentido, é relevante identificar e discutir a exposição dos profissionais à violência ocupacional e as formas de enfrentamento individual, coletiva e organizacional na RAS, uma vez que os serviços que compõem a rede são preferencialmente localizados em áreas de maior vulnerabilidade social ${ }^{8}$. A violência ocupacional além de interferir na dinâmica do atendimento, amplia as taxas de absenteísmo por motivos diversos, rotatividade dos profissionais e problemas de saúde física e mental ${ }^{9}$.

Com o intuito de subsidiar o presente estudo, realizou-se a revisão integrativa da literatura acerca da violência ocupacional na ESF tendo como base a Biblioteca Virtual da Saúde (BVS) no modo integrado com a Base de Dados de Enfermagem (BDENF), Literatura Latino-Americana e do Caribe em Ciências da Saúde (LILACS), Medical Literature Analysis and Retrieval System Online (MEDLINE) e na Cumulative Index to Nursing and Allied Health Literature (CINAHL). Selecionados artigos publicados prioritariamente no país no período de 2014 a 2018. Entre as 96 produções acessadas, excetuando os artigos duplicados, os de revisão e reflexão, a revisão demonstrou que apenas 12 produções enfrentam centralmente o debate sobre a violência ocupacional e suas relações com a área de saúde. A incipiência na produção de conhecimento sobre o tema fica ainda mais evidente quando focalizada a ESF, o que justifica a necessidade de aprofundar a análise sobre este objeto.

O presente estudo tem por objetivos: identificar a ocorrência da violência na Estratégia de Saúde da Família e analisar as repercussões para a saúde dos trabalhadores e o acolhimento.

\section{REVISÃO DA LITERATURA}

A violência na atualidade é um problema de saúde pública que afeta indivíduo e coletividade independente do credo, raça, cor, idade, sexo e poder aquisitivo com sérias repercussões para o indivíduo e a sociedade em geral devido a encargos emocionais, sociais e econômicas. Daí a importância da prevenção da violência em todos os espaços sociais como família, escola e trabalho ${ }^{10}$. A Política Nacional de Redução da Morbimortalidade por Acidentes e Violência, ao reconhecer a violência como um problema de saúde pública, norteia a atuação dos serviços de Saúde neste contexto ao assumir a violência como "evento representado por ações realizadas por indivíduos, grupos, classes ou nações que ocasionam danos físicos, emocionais, morais e/ou espirituais a si próprio ou a outros ${ }^{11: 7}$."

No ambiente de trabalho a violência é reconhecida como qualquer ação, incidente ou comportamento baseado em uma atitude instintiva do agressor, em consequência da qual um profissional é agredido, ameaçado, sofre algum dano ou lesão durante a realização do trabalho ${ }^{12}$. No final da década de 1990, observou-se um aumento da preocupação com a violência ocupacional, quando no campo da Saúde do Trabalhador, os olhares também se voltaram para o problema. Neste período a Organização Internacional do Trabalho (OIT) e a Organização Mundial de Saúde (OMS) passaram a realizar os primeiros esforços no sentido discutir este evento e propor ações conjuntas no seu enfrentamento. A partir deste marco, a literatura tem dispensado mais atenção ao problema e algumas instituições passaram a mensurar este fenômeno e suas repercussões para a organização e a saude do trabalhador ${ }^{13}$.

Atualmente rejeita-se a ideia de que a violência ocupacional comporte fatores unicamente pessoais e passa-se a considerá-la como resultante de uma combinação de causas relativas às pessoas, ao entorno, ao ambiente físico e social do trabalho e às condições organizacionais e contratuais de emprego, bem como as formas de interação entre os próprios trabalhadores, entre os clientes e os trabalhadores e entre esses e empresários 5 .

\section{MÉTOdo}

Trata-se de um estudo qualitativo descritivo e exploratório. O campo foi uma unidade da ESF inaugurada em 2011, localizada no município do Rio de Janeiro que possui aproximadamente 10.133 usuários cadastrados. As atividades de cunho técnico e assistencial são realizadas por cinco equipes de saúde. A unidade funciona de segunda à sexta-feira das 7 às 18 horas e aos sábados das 8 às 12 horas, havendo, neste dia, revezamento entre as equipes. A ESF faz parte da rede de 
serviços da Área Programática 3.2 (AP 3.2) administrada por uma Organização Social de Saúde (OSS), abrangendo 23 bairros da zona norte. Integra a ESF o Núcleo de Apoio à Saúde da Família (NASF) composta por uma equipe complementar composta de profissionais da saúde e de diferentes ocupações, de maneira integrada para dar suporte (clínico, sanitário e pedagógico) aos profissionais da ESF, de modo a garantir a continuidade de cuidados à população.

O projeto foi aprovado pelo Comitê de Ética em Pesquisa da instituição envolvida (Parecer no 2.668.601/SMS-RJ). Em atendimento à Resolução 466/12, após explicações sobre os objetivos e demais aspectos éticos os participantes assinaram o Termo de Consentimento Livre e Esclarecido. Participaram do estudo 27 trabalhadores da saúde (13 agentes comunitários de saúde, 2 auxiliares de saúde bucal, 3 enfermeiros, 4 médicos, 4 técnicos de enfermagem, 1 técnico de saúde bucal). Na seleção dos participantes, trabalhou-se com os seguintes critérios de inclusão: trabalhadores com pelo menos um ano de atuação na ESF e em pleno exercício de suas atividades profissionais na ocasião em que os dados foram coletados. Excluídos os trabalhadores afastados devido a problemas de saúde, férias e licenças de outra natureza.

Informou-se que a participação seria voluntária e que os participantes poderiam se retirar da pesquisa em qualquer fase, sem que houvesse qualquer dano ou retaliação. Garantiu-se o anonimato e ratificou-se que os resultados seriam apresentados em eventos e publicados em revistas científicas. Na transcrição dos depoimentos adotou-se as seguintes convenções: ACS (Agente Comunitário de Saúde), ASB (Auxiliar de Saúde Bucal), ENF (Enfermeiro), MED (Médico), TE (Técnico de Enfermagem), TSB (Técnico de Saúde Bucal) seguido de um número de acordo com a ordem de realização das entrevistas.

A coleta de dados foi realizada no primeiro semestre de 2018 , individualmente, em local privativo e livre de interferências após agendamento e disponibilidade dos entrevistados, tendo durado em torno de 30 minutos. Optouse pela técnica de entrevista semiestruturada mediante um roteiro com questões abertas. Na caracterização dos participantes foi utilizado um instrumento estruturado.

Realizada a transcrição, os depoimentos foram analisados mediante a técnica de análise de conteúdo do tipo temática ${ }^{14}$ que se baseou na decodificação do texto em diversos elementos, os quais foram classificados e formaram agrupamentos analógicos. Em um último momento, utilizando os critérios de representatividade, homogeneidade, reclassificação e agregação dos elementos do conjunto, chegou-se aos seguintes eixos temáticos: violência psicológica perpetrada pelos usuários e violência relacionada ao trabalho no território.

\section{RESULTADOS E DISCUSSÃo}

\section{Violência psicológica perpetrada pelos usuários}

Os profissionais de saúde que atuam na ESF possuem relevante papel junto à população no que diz respeito ao trabalho de educação em saúde, vigilância epidemiológica, vacinação e demais aspectos voltados para a prevenção de agravos à saúde. Neste sentido, cabe a reflexão sobre o fluxo de atendimento das famílias cadastradas na unidade e daqueles que procuram o serviço com demandas que nem sempre são compatíveis com o serviço. O estresse do usuário decorrente da falta de resolutividade em relação ao atendimento contribui para os quadros de violência psicológica perpetrada contra os profissionais como referido:

O serviço não funciona! Quem fica no meio disso é o profissional. Tem paciente que vem aqui várias vezes e não resolve seu problema. Chega uma hora que a pessoa perde a cabeça. Colocam a culpa na gente! Partem pra cima xingando a gente, batendo na mesa, ameaçando. Todo dia é assim (E10TE).

Virou rotina! Você ver seu colega ser xingado no ambiente de trabalho ou sofrer ameaça. Essas coisas acontecem com tanta frequência hoje em dia, que ficou normal (E3TE).

Tem alguns pacientes que se descontrolam. Tem funcionários aqui que já foram agredidos tanto verbal como tentaram agredir fisicamente (E17ACS).

Tais relatos além de exporem a problemática da falta de resolutividade dos problemas de ordem técnica e assistencial, perpassam as representações que os trabalhadores possuem sobre o perfil dos pacientes agressivos, que por se mostrarem descontrolados passam ser objeto da assistência, exigindo habilidades para o enfrentamento da violência e refletem as relações de poder no espaço social do trabalho ${ }^{4}$. Por sua vez, devem-se considerar atributos como longitudinalidade, abrangência da integralidade, orientação para comunidade e competência cultural dos profissionais, pois na medida em que são concretizados no dia a dia do trabalho transformam a visão da violência e suas causas como um preocupante determinante social de saúde e que exige intervenções planejadas, de cunho intersetorial e com a participação da comunidade ${ }^{7}$.

Ratifica-se a relevância da capacitação e sensibilização dos profissionais para a prevenção da violência no trabalho. A qualificação possibilita a construção de vínculos mais próximos ao usuário e o desenvolvimento de uma escuta qualificada com vistas ao encaminhamento ou agendamento efetivo ${ }^{3}$. Conhecer a relação existente entre o acesso e a 
satisfação do usuário possibilita alternativas para a melhoria da qualidade dos serviços em saúde, devendo-se considerar aspectos relacionados ao fluxo de atendimento, tempo despendido na sala de espera e a satisfação com o agendamento, consultas e exames. Portanto, cabe ao gestor avaliar a necessidade de investimentos em infraestrutura e recursos humanos adequados à demanda².

A postura dos usuários na ânsia por uma assistência em saúde rápida e resolutiva ou ainda, o medo dos profissionais devido à violência nas áreas de cobertura da ESF, gera muitas vezes insatisfação e sofrimento, uma vez que o trabalho na ESF exige o estabelecimento de relações congruentes para que a assistência em saúde consiga ser mais resolutiva ${ }^{9}$. Assim, a violência no trabalho se destaca como um relevante problema de saúde coletiva na contemporaneidade e, apesar de subnotificada ganha atenção frente às repercussões para a saúde dos profissionais e no atendimento dos usuários ${ }^{7}$.

Como constado no presente estudo, existe certa naturalização e até mesmo banalização da violência psicológica no trabalho. A não ser que ocorra um episódio considerado grave ou surjam ameaças contínuas, procedimentos formais de notificação são pouco conhecidos e utilizados pelos profissionais. Acredita-se que uma política de não valorização do profissional da saúde aliada a uma sociedade em que não há punição para os atos de violência acabam por desmotivar a denúncia e a busca por providências.

Diante da problemática apontada, ratifica-se a relevância da participação do trabalhador e da comunidade na elaboração de projetos voltados para a prevenção, monitoramento e combate da violência mediante notificação da sua ocorrência, educação e/ou treinamento das equipes e adoção de medidas que garantam a segurança das equipes na unidade e/ou no território durante as visitas domiciliares e demais atividades técnicas ${ }^{15}$.

No entanto, constata-se que ainda é frágil a cultura institucional nas equipes de saúde da família em notificar a violência sofrida, fato que dificulta ainda mais a proposição de estratégias para seu enfrentamento efetivo. Os trabalhadores de saúde apresentam pouco interesse em notificar os casos de violência por desconhecerem seus desdobramentos e por considerarem que seus resultados são de pouca utilidade na realidade do trabalho ${ }^{16,17}$. Contudo, a não informação dos casos de violência vivenciados pelos profissionais independente de sua natureza e das relações entre os envolvidos, acarreta a perpetuação cíclica do problema, impossibilitando a formulação de estratégias efetivas para a contenção dessa problematica ${ }^{9,11,15}$.

\section{Violência relacionada ao trabalho no território}

Desde a implantação da ESF na década de 1990, a prerrogativa foi iniciar a cobertura assistencial nas áreas consideradas de maior vulnerabilidade social. Além desta questão, na organização do processo de trabalho das equipes é previsto um relacionamento mais próximo à população adstrita, cuja assistência é realizada usualmente em ambientes abertos ou na própria residência dos usuários ${ }^{16}$. A violência nestas áreas e a decorrente do tráfico de drogas, além de dificultarem a continuidade de serviços essenciais, geram nos profissionais sentimento de insegurança e de imprevisibilidade pois, a qualquer momento podem eclodir situações de violência com riscos para a integridade física e mental dos trabalhadores como identificado:

Você está lá dentro (referindo-se à comunidade) e pode sair tiroteio! Você não tem onde se esconder! Sei lá! Pode acontecer coisa pior! Eu acho bem violento! Bem perigoso (E19TE).

A gente passa na frente da boca de fumo! Então tem menino novo, pequeno e tal com arma! É bem pesado, mas a gente acostuma (E26MED).

Eu fui assaltado aqui na frente! Levaram meu carro! Fiquei refém com arma na cabeça. Levaram tudo meu! Foi um momento bem difícil! Traumático (E1MED).

A violência como barreira de acesso se refere a uma realidade constante no cotidiano das comunidades. As regras de acesso impostas por facções ligadas ao tráfico de drogas e armas inibem a entrada de profissionais de saúde que não moram nas comunidades, comprometendo a visita domiciliar das equipes de ESF e ao mesmo tempo prejudicam a circulação de alguns moradores até o território onde a UBS está instalada. É possível, ainda, observar que situações de violência geram uma maior rotatividade dos profissionais, acarretando descontinuidade nos processos de trabalho e enfraquecimento dos vínculos com a comunidade ${ }^{7}$.

Existem relações simétricas entre a violência, a estrutura social, econômica e política de um país aprofundandose com as exclusões e desproteções sociais; características do atual modelo neoliberal gerador de insegurança de toda ordem. Inclui-se nesse contexto a atenção e a promoção à saúde em áreas vulneráveis à violência, comumente caracterizadas por uma infraestrutura precária, com privação de condições materiais de vida e profundas desigualdades sociais. Além das questões sociais, as pessoas que residem em áreas consideradas de risco vivenciam situações de exclusão, a exemplo do desemprego, da falta de saneamento básico e da escassez de lazer e segurança ${ }^{10}$. 
As implicações da violência para a saúde dos trabalhadores foram identificadas a partir de expressões de sofrimento que perpassaram o medo e a insegurança diante de riscos para integridade física e psíquica. Como referido, o atendimento dos usuários é prejudicado em função do sentimento de desproteção e medo:

Me afeta muito psicologicamente. A gente já vem com medo! Medo de chegar à unidade, medo de fazer visita, medo de ir pra casa. É assim o tempo todo, o tempo todo esse sentimento (E5ENF).

Ah, a gente fica com muito medo, muito inseguro e isso interfere diretamente no nosso atendimento. $O$ atendimento não é o mesmo. Acho que a gente fica muito exposta! Sem proteção. Isso me deixa muito pensativa em relação a esse trabalho (E2OTSB).

Acho que o profissional aqui se sente muito, muito inseguro, com muito medo, diariamente! Já teve situações aqui do profissional não saber mais como voltar ao equilíbrio pra poder dar conta de uma consulta (E11ENF).

A violência urbana tem afetado a adequação dos serviços à realidade local, o que pode representar inúmeros empecilhos para a promoção efetiva da assistência em saúde por parte das equipes. São limitações no acesso à unidade de saúde e na realização das visitas domiciliares que inviabilizam atividades como grupos educativos, consultas e outras ações de prevenção e promoção da saúde. Há também problemas relativos à insegurança, infraestrutura inadequada, convívio com o tráfico de drogas, entre outros fatores, que tornam o cuidado com a saúde um desafio diário ${ }^{8,18}$.

A violência, e nela incluído o comércio ilegal de drogas, além de gerar prejuízos para a qualidade de vida das pessoas constitui uma grave ameaça à integridade física e psíquica dos envolvidos. O comércio ilegal de drogas é preponderante para a ocorrência da violência e condiciona inúmeras situações de conflito entre traficantes e usuários, acarretando o aumento da criminalidade, mortes preveníveis e interrupção da prestação de serviços essenciais a população ${ }^{6}$.

Para os profissionais de saúde, a ausência de segurança no território, além de gerar medo e restringir o acesso ao local de trabalho, interfere na realização das atividades, com repercussões para a subjetividade do trabalhador, sua satisfação e motivação. Há também o sofrimento psíquico identificado através de expressões de medo, insegurança e ansiedade ${ }^{9}$.

Os profissionais enfrentam uma série de desafios que limitam sua prática profissional como o medo do confronto entre as comunidades rivais, o convívio com usuários de drogas ilícitas e com o tráfico. Entre as limitações assistenciais impostas pela vulnerabilidade à violência, estão: a dificuldade de acesso aos domicílios, locomoção das equipes, a fragilidade diante de ameaças físicas, a luta para conseguir a confiança da população e a diminuição do comparecimento da população às unidades 5 .

\section{Limitações do estudo}

Apesar das limitações do estudo em decorrência do método, do número de participantes e por ter sido realizado em uma única unidade da ESF, o que impossibilita a generalização dos resultados para outros contextos de trabalho, ratifica-se a sua relevância ao discutir a violência urbana e no território e aspectos voltados para a notificação e o treinamento das equipes no intuito de qualificar o atendimento e minimizar a sua ocorrência.

\section{CONCLUSÃO}

Conclui-se que trabalhadores da ESF encontram-se expostos à violência psicológica perpetrada pelos usuários em forma de ameaça verbal, insultos e intimidação, devido à insatisfação com o atendimento e a não resolutividade dos problemas. Esses atos além de interferirem na qualidade do atendimento constituem riscos para a integridade física e psíquica dos trabalhadores.

A violência urbana ou de trajeto foi identificada diante da exposição dos profissionais ao tráfico de drogas, tiros, policiamento e assaltos, pelo fato de a unidade da ESF estar localizada em área de maior vulnerabilidade social.

A violência interfere negativamente no trabalho dos profissionais e no que diz respeito às demais atividades práticas voltadas para a prevenção, promoção e educação em saúde.

\section{REFERÊNCIAS}

1. Coutinho LRP, Barbieri AR, Santos LMM. Reception in Primary Health Care: an integrative review. Saúde Debate [Internet]. 2015 [cited 2019 Aug 23]; 39(105):514-24. DOI: http://dx.doi.org/10.1590/0103-110420151050002018.

2. Carlos LI, Costa JF, Lima Filho BF, Guedes MBOGG. Reception and family health strategy: experience report. Rev. Med. Minas Gerais. [Internet]. 2017 [cited 2019 Sep 21]; 27: e-1916. DOI: http://dx.doi.org/10.5935/2238-3182.20170095.

3. Lira LBS, Santos DS, Neves SJF, Nagliate PC, Pereira EAT, Cavalcante MV. Acess, wellcoming and Family health strategy: user satisfaction. Rev enferm UFPE. [Internet] 2018 [cited 2019 Sep 12]; 12(9):2334-40. DOI: https://doi.org/10.5205/1981-8963v12i9a234878p2334-2340-2018. 
4. Dal Pai D, Sturbelle ICS, Santos C, Tavares JP, Lautert L. Physical and psychological violence in the workplace of healthcare professionals. Texto contexto-enferm. [Internet] 2018 [cited 2019 Sep 12]; 27(1):e2420016. Available from: DOI: http://dx.doi.org/10.1590/0104-07072018002420016.

5. Santos MS, Silva JG, Branco JGO. Fighting violence under the family health strategy: challenges for health care. Rev. Bras. Promoç. Saúde. [Internet] 2017 [cited 2019 Sep 12]; 30(2):229-38. Available from: https://periodicos.unifor.br/RBPS/article/view/5895/pdf.

6. Lopes CS, Moraes CL, Junger WL, Werneck GL, Leon ACP, Faerstein E. Direct and indirect exposure to violence and psychological distress among civil servants in Rio de Janeiro, Brazil: a prospective cohort study. BMC Psychiatry. [Internet] 2015 [cited 2019 Aug 23]; 15(109):1-9. DOI: https://doi.org/10.1186/s12888-015-0487-9.

7. Benificio LSF, Barros JPP. Strategy family health and urban violence: social approaches and practices in question. SANARE Sobral. [Internet] 2017 [cited 2019 Sep 12]; 16 (spl):102-12. Available from: https://sanare.emnuvens.com.br/sanare/article/view/1146.

8. Machado CB, Daher, DV, Teixeira ER, Acioli S. Urban violence and effect on care practices in family health strategy territories. Rev. enferm. UERJ. [Internet] 2016 [cited 2019 Sep 15]; 24(5):e25458. DOI: http://dx.doi.org/10.12957/reuerj.2016.25458.

9. Soratto J, Pires DEP, Trindade LL, Oliveira JSA, Forte ECN, Melo TP. Job dissatisfaction among health professionals working in the family health strategy. Texto contexto-enferm. [Internet] 2017 [cited 2019 Aug 23]; 26(3):e2500016. DOI: http://dx.doi.org/10.1590/0104-07072017002500016.

10. Minayo MCS. A violência dramatiza causas. In: Minayo MC, Souza ER, organizadores. Violência sob o olhar da saúde: a infrapolítica da contemporaneidade brasileira. Rio de Janeiro. Fiocruz; 2011.

11. Ministério da Saúde (Br). Política Nacional de Redução da Morbimortalidade por Acidentes e Violência : Portaria MS/GM no 737 de 16/5/01, publicada no DOU no 96 seção 1e, de 18/5/01 / Ministério da Saúde. Brasília (DF): Ministério da Saúde, $2002 ; 64$ p. [cited 2019 Aug 23]. Available from: https://pesquisa.bvsalud.org/bvsms/resource/pt/mis-249.

12. Organización Internacional del Trabajo, Consejo Internacional de Enfermeras, Organización Mundial de la Salud, Internacional de Servicios Públicos. Directrices marco para afrontar la violencia laboral en el Sector de la Salud [Internet]. Ginebra: OIT; 2002 [cited 2019 Sep 12]. Available from: https://apps.who.int/iris/bitstream/handle/10665/44072/9223134463_spa.pdf?sequence=1\&isAllowed=y.

13. Almeida NR, Bezerra Filho JG, Marques LA. Analysis of the scientific production on violence at work in hospital services. Rev. Bras. Med. Trab. [Internet] 2017 [cited 10 Aug 2019]; 15(1):101-12. Available from: http://www.rbmt.org.br/details/218/pt.

14. Bardin L. Análise de Conteúdo. Tradução de Luis Antero Reto e Augusto Pinheiro. São Paulo: Edições 70/Livraria Martins Fontes; 2011.

15. Oliveira LP, Camargo FC, Iwamoto HH. Violence related to the work of family health teams. REAS. [Internet] 2013 [cited 2019 Aug 10]; 2(2):46-56. Available from: http://seer.uftm.edu.br/revistaeletronica/index.php/enfer/article/view/388/417.

16. Silva IV, Aquino EML, Pinto ICM. Violência no trabalho em saúde: a experiência de servidores estaduais da saúde no Estado da Bahia, Brasil. Cad. Saúde Pública. [Internet] 2014 [cited 2019 Sep 12]; 30(10): 2112-22. Available from: http://www.scielo.br/scielo.php?script=sci_arttext\&pid=S0102.

17. Fontes KB, Santana RG, Pelloso SM, Carvalho MDB. Factors associated with bullying at nurses' workplaces. Rev. Latino-Am. Enferm. [Internet] 2013 [cited 2019 Aug 10]; 21(3):758-64. Available from: http://www.scielo.br/pdf/rlae/v21n3/pt_0104-1169rlae-21-03-0758.pdf.

18. Polaro SHI, Gonçalves LHT, Alvarez AM. Nurses challenging violence in the scope of practice in the Family Health Program. Texto context-enferm. [Internet] 2013 [cited 2019 Aug 23]; 22(4):935-42. DOI: http://dx.doi.org/10.1590/S0104-07072013000400009.

19. Melquiades DD, Leite MCA. O estresse ocupacional em equipes de saúde da família. Rebes. Revista Brasileira de Educação em Saúde. [Internet] 2014 [cited 2019 Sep 12]; 4(2): 27-32. Available from: https://gvaa.com.br/revista/index.php/REBES/article/viewFile/3051/2538. 